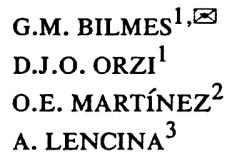

\section{A real time method for surface cleanliness measurement}

\author{
${ }^{1}$ Centro de Investigaciones Opticas (CONICET-CIC) and Universidad Nacional de La Plata, \\ La Plata, Argentina \\ 2 Departamento de Física, (FCEyN-Universidad de Buenos Aires) and CONICET, Bs. As, Argentina \\ ${ }^{3}$ Laboratório de Óptica Não Linear, Departamento de Física, Universidade Federal da Paraíba \\ João Pessoa, Brazil
}

\begin{abstract}
Received: 13 July 2005/Revised version: 29 September 2005 Published online: 18 January 2006 • @ C Springer-Verlag 2005

ABSTRACT The measurement of surface cleanliness is a signifcant problem in many industrial and technological processes Existing methods are based on laboratory procedures, that are not performed in real time, can not be automated, and usually are restricted to a small portion of the sample. In this study we describe a new method for real time measurement of the amount of surface dirt or contamination deposited on a surface. It relies on the ablation of the surface dirt film by means of a short laser pulse, and the subsequent measurement of the emitted sound. The intensity of the sound is proportional to the amount of surface dirt and provides a direct measurement of the cleanliness of the surface. We also developed a reference sample for calibration, based on a uniform distribution of dots printed on white paper. The density and the dot size can be easily modified providing a homogeneous, uniform and reproducible standard for the measurement. Based on this method, we designed, developed and patented the first industrial instrument for on-line determination of the degree of cleanliness of manufactured cold rolled steel plate bobbins.
\end{abstract}

PACS 79.20.D; 81.70.C; 42.62.

\section{1}

\section{Introduction}

The measurement of the levels of cleanliness on a surface is a problem of great importance in many industrial and technological processes and in scientific research (fiber optics, plastics, airplane and siderurgical industries, the conservation of pieces of cultural value, in material science, etc.). Usually the main problems are to define what level of cleaning of the surface is necessary for a certain quality standard and how to measure it [1]. Surface dirt deposited on an object may have different origins. It may come from handling, aging, pollution, or could be a consequence of fabrication or industrial procedures. In many cases surface dirt can be described as a dark thin film of organic substances such as oil or grease in which a more or less homogeneous distribution of particles of different compounds is present. Most of these particles are black, giving the dirt a dark aspect. In other cases like in some "clean industries" such as microelectronics, micromechanics,

Fax: 54221471 2771, E-mail: gabrielb@ciop.unlp.edu.ar data storage, etc. the main dirt is usually composed of small isolated particles added to the substrate.

Laser cleaning methods have been developed over the last twenty years, including patents and a theoretical understanding of the mechanisms involved [2-7].

Existing methods for surface cleanliness determination of dirt measurements are based, in most of the cases, on laboratory procedures, outside production lines $[8,9]$, and in special cases they give a direct measurement [10]. Many of them rely on the quantitative analysis of the residues removed by solvent extraction (i.e. by washing) or burned from the surface, or the measurement of the transparency of an adhesive tape that has been stuck and removed from the surface to be tested (tape method) [11].

All these methods have the following drawbacks: they are not performed in real time, and are time consuming; they cannot be automated, and usually they are restricted to a small portion of the sample.

In this study a new method that overcomes all the above mentioned drawbacks is described [12]. The method allows real time measurement of the relative or absolute amount of dirt films present on different types of surfaces. It relies on the ablation of the dirt film by means of a short laser pulse and the subsequent measurement of the emitted sound. The intensity of the sound is proportional to the amount of dirt and provides a direct measurement of the cleanliness of the surface. For the case of cold rolled steel plates, in a previous work we showed that there is an excellent correlation between the acoustic measurements and the standard laboratory methods [13, 14].

Surface dirt can be found on any object, but there is no simple way to produce it in controlled conditions. Therefore, most of the laboratory methods that measure the cleanliness of a surface use an indirect calibration reference. To our knowledge, there are no references samples for calibration purposes, of controlled thickness, particle size, and homogeneity of the dirt distribution. For that reason we developed a dirt ref= erence for calibration purposes based on a uniform distribution of dots printed on white paper. The density of points and their size can be easily modified by a simple computer program, providing a homogeneous, uniform and reproducible standard for dirt measurements.

Based on the method presented above, we designed and patented a commercial instrument named ELMES (Equipo 
Láser para Medición de Suciedad) for industrial purposes, that can measure on-line the cleanliness of surfaces [13-16]. The first prototype of this instrument was developed for the siderurgical industry, and was used for the determination of the amount of residual dirt on steel plates after cold rolling. This information is crucial, both for the effectiveness of downstream operations such as galvanizing or painting and as a control of possible malfunctioning of prior stages of the process. This equipment was mounted at the final inspection line of the Ensenada plant of the company SIDERAR of Argentina.

\section{Experimental}

A schematic of the set up used is shown in Fig. 1. A Q-switched Nd: YAG laser with pulse duration of c.a. $7 \mathrm{~ns}$ (FWHM) operating at a wavelength of $1064 \mathrm{~nm}$ is directed normal to the sample surface. A neutral density wedge filter is used to change the energy of the laser pulse to produce laser fluences (energy per unit area), $F$, ranging from $0<F<4.5 \mathrm{~J} / \mathrm{cm}^{2}$. For measurements as a function of the excitation area, we change the fluence by modifying the diameter of the laser beam using a focusing lens. A microphone picks up the emitted noise. The detected acoustic signal is registered on an oscilloscope and is correlated with the amount of dirt present on the sample. The pulse energy was measured using an energy meter with a pyroelectric detector, splitting the laser pulse by means of a calibrated beam splitter. Taking into account the non-homogeneous spatial energy distribution of the laser pulse and then the effective area of the laser spot, the fluence values calculated in this work have an estimated uncertainty of c.a. $30 \%$.

The sample can be moved in order to avoid overlapping of successive laser shots. Then, the whole sample can be analyzed giving a statistical measure of the dirt distribution on the surface. It means that not only the mean value, but also its dispersion can be tested. In this way the homogeneity of the surface dirt can be evaluated. When necessary, averaging of several shots, impinging on different sites of a small region of the sample, can be made in order to further increase the signal to noise ratio and subtract background noise.

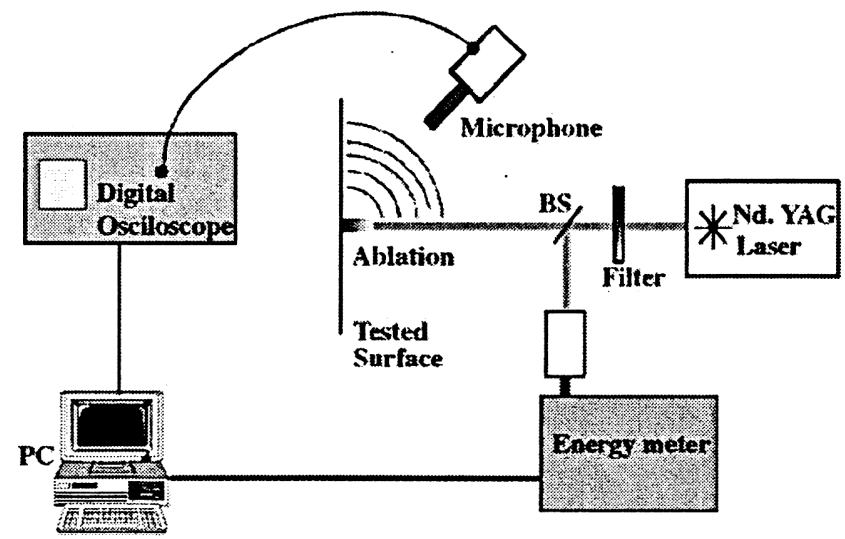

FIGURE 1 Experimental set-up used for real time cleanliness measurements
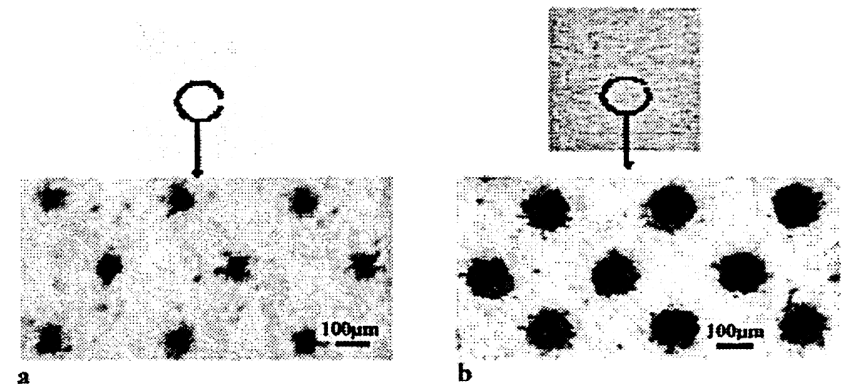

FIGURE 2 Bare eye (upper) aspect and microscopic detailed picture (lower) of the reference samples used, which are composed by uniform, homogeneous and reproducible distributions of ink points on white paper. (a) Point diameter $\Phi=60 \pm 10 \mu \mathrm{m}$. (b) Point diameter $\Phi=110 \pm 10 \mu \mathrm{m}$

Different types of surface dirt were analyzed: dirt produced by hand=manipulation of plastic surfaces; residuals generated during manufacturing of cold rolled steel plates and candle soot deposited on glass substrates.

For opaque samples, such as steel plates, conventional measurements were performed using the tape method. In this case a transparent adhesive tape is stuck to the sample and removed, containing the surface dirt. Afterwards, the tape is stuck to a white paper and the transparency is measured by reflectometry. For transparent substrates (i.e. glass) the transmission of a He-Ne cw laser beam was measured before the ablation of the dirt.

Since it is not possible to produce surface dirt with controlled thickness, size and distribution of the particles that compose the dirt, we decided to develop reference samples. These dirt references can be made easily by means of a simple computer program and a printer. They are composed of a uniform distribution of black ink dots printed on white paper. We used a standard graphic computer program (Corel 9.0) and a HP LaserJet 5100 printer that allow us to change the amount of surface points per square inch $\left(\mathrm{p} / \mathrm{inch}^{2}\right)$ and the size of the dots, keeping constant the thickness of the distributed ink in the paper. For the experiments presented here we used uniform, homogeneous and reproducible distributions of ink points of two different measured diameters of $\Phi=$ $60 \pm 10 \mu \mathrm{m}$ and $\Phi=110 \pm 10 \mu \mathrm{m}$, in sets of samples with points densities of : $800 \mathrm{p} /$ inch $^{2}, 1600 \mathrm{p} / \mathrm{inch}^{2}, 3200 \mathrm{p} / \mathrm{inch}^{2}$, $6400 \mathrm{p} /$ inch $^{2}$ and $12800 \mathrm{p} /$ inch $^{2}$.

Figure 2 shows a bare eye and a microscopic detailed picture of the reference samples used.

\section{Results and discussion}

When a laser pulse of suitable fluence and short du= ration (several $\mathrm{ns}$ ) impinges on a surface, ablation of the dirt film takes place without damaging the substrate. During ab= lation, a layer of the dirt film is abruptly expelled from the surface, a weak light pulse is emitted, and a sound that sounds like a 'crack' is produced. When the sound is detected by means of a microphone set at a fixed distance and angle with respect to the impact zone of the plate, a typical signal can be measured as shown in Fig. 3. This figure shows results cor= responding to the ablation of a plastic surface with different degrees of cleanliness. As it can be seen the intensity of the sound depends on the amount of dirt and provides a direct 


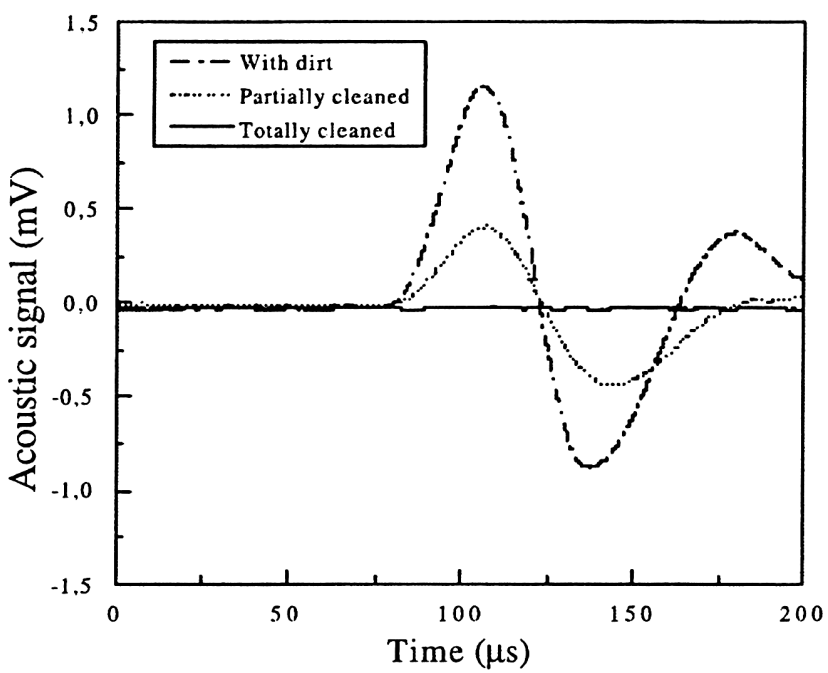

FIGURE 3 Amplitude of the acoustic signals detected by a microphone, corresponding to the ablation of dirt from a plastic surface with different degrees of cleanliness

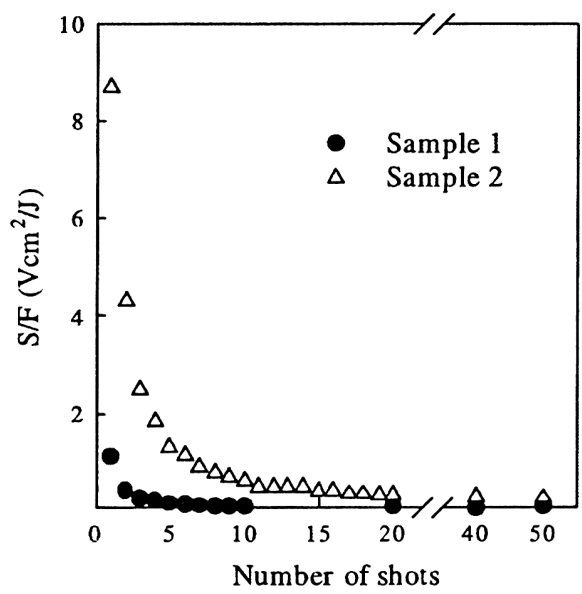

FIGURE 4 Acoustic signal $S$ normalized in fluence $F$ as a function of the number of shots impinging on the same point of the sample. Sample 2 is dirtier than sample 1

measurement of the cleanliness of the surface. We then used the peak-to-peak voltage of the acoustic signal detected by the microphone as a measure of the amount of dirt.

Figure 4 shows the dependence of the peak to peak value of the detected acoustic signal $S$ divided by the laser fluence $F$ as a function of the number of laser shots that impinges on the same point of the surface, for two different samples with different amount of dirt. Sample 2 is dirtier than the sample 1. As can be seen, dirtier samples yield larger values for $S / F$, and more shots are necessary to clean the surface.

In order to optimize the detection conditions, we studied the response of the microphone as a function of its angular position, performing measurements in two planes perpendicular to the surface. We found a more or less spherical distribution of the emission of sound, which follows the inverse square law. This allows high flexibility for reception designs, provided that the calibration is performed at the final set up configuration.

Taking into account this result and the need in some applications to avoid possible effects of environmental noise, we

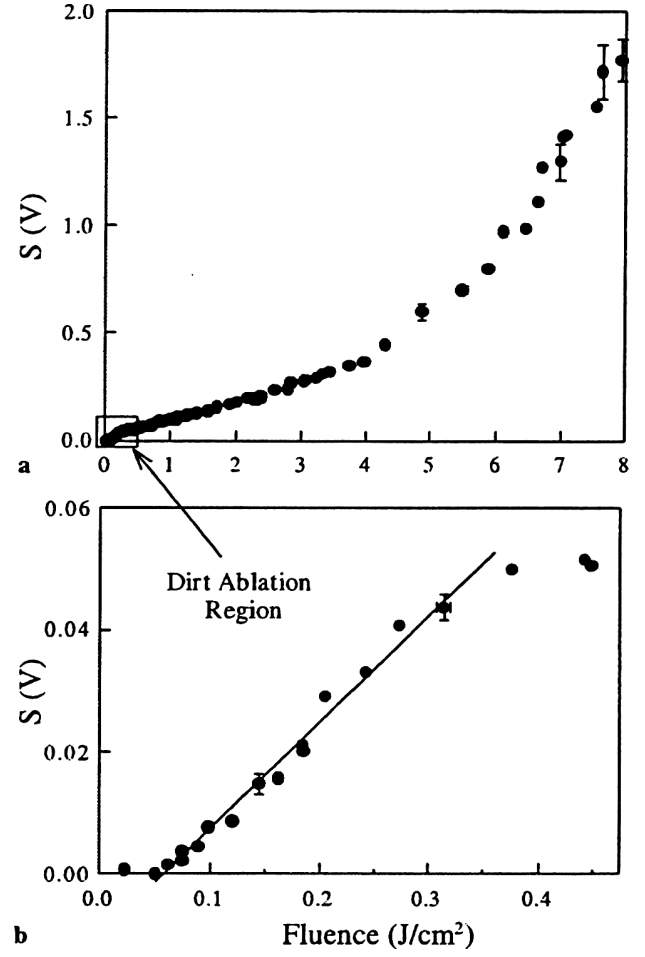

FIGURE 5 (a) peak to peak amplitude of the detected acoustic signal as a function of the laser fluence for a steel plate with dirt residuals generated during manufacturing processes. (b) Shows a detail of the low fluence part of the curve corresponding to the dirt ablation region in which there is no damage of the substrate. An error bar in some measurements corresponds to average (10) values and its uncertainty

improved the directionality of the detection, by mounting the microphone at the end of a small $5 \mathrm{~cm}$ long plastic tube .

\subsection{Threshold fluences for dirt ablation and substrate damage}

As is usual in any ablation process, there is a fluence threshold below which there is neither ablation of the dirt nor a detectable acoustic signal. Above this threshold value, ablation of the dirt film occurs and acoustic signals are emitted. For larger values of the fluence, ablation of the substrate can also take place. For a correct measurement of the amount of dirt any influence of the substrate has to be avoided. For that purpose, the laser fluence must be sufficient to produce the ablation of the dirt, but it should be below the threshold for ablation and damage of the substrate.

Figure 5a shows the typical dependence of the detected acoustic signal $S$ as a function of the laser fluence for a dirty surface and Fig. 5b is a detail of the low fluences region. As can be seen, below $0.05 \mathrm{~J} / \mathrm{cm}^{2}$ there is no acoustic signal. Between 0.05 and $0.3 \mathrm{~J} / \mathrm{cm}^{2}$ the acoustic signal grows linearly with the laser fluence and above $0.3 \mathrm{~J} / \mathrm{cm}^{2}$ a plateau is reached. At even higher fluences a new linear region appears between c.a. 1 and $4 \mathrm{~J} / \mathrm{cm}^{2}$. On increasing the fluence a new slope change appears and above $5 \mathrm{~J} / \mathrm{cm}^{2}$ a new linear region is obtained.

Figure 6 shows the dependence of the detected acoustic signal as a function of the number of laser shots. Each curve 


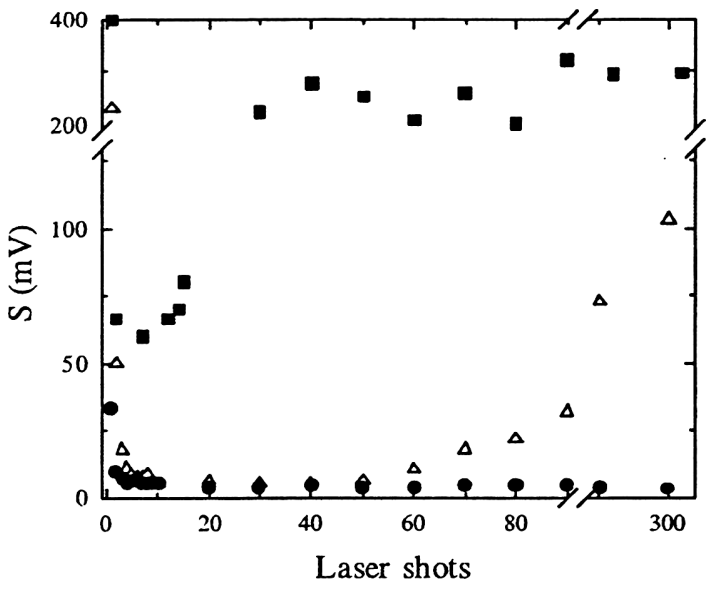

FIGURE 6 Peak to Peak detected acoustic signal as a function of the number of laser shots that impinges on the same point of the sample. $(\bullet) F=0.2 \mathrm{~J} / \mathrm{cm}^{2} ;(\Delta) F=3 \mathrm{~J} / \mathrm{cm}^{2} ;(\square) F=7 \mathrm{~J} / \mathrm{cm}^{2}$ (values are divided by 3 to fit the plot). Sample: steel plate with dirt residuals generated during manufacturing process

corresponds to laser shots of the same fluence, impinging in a fixed area of the sample. It can be seen that for a fluence of $0.2 \mathrm{~J} / \mathrm{cm}^{2}$, after a certain number of laser shots no acoustic signal can be detected. At $3 \mathrm{~J} / \mathrm{cm}^{2}$ the acoustic signal corresponding to the first shot is higher than that for $0.2 \mathrm{~J} / \mathrm{cm}^{2}$ and after that, the acoustic signal decreases from shot to shot but, instead of disappearing, after ca. 50 shots it increases. At fluences of $7 \mathrm{~J} / \mathrm{cm}^{2}$, a higher intensity of the acoustic signal is observed for the first shot, and after that, $S$ tends to rapidly reach a constant value.

The behavior shown in Figs. 5 and 6 was found for the different types of dirt samples and substrates studied in this work. It is as expected for an ablation processes and is in very good correlation with time-integrated light intensity plasma emission measurements [17]. We can interpret these results as follows: at lower fluences no surface ablation takes place. Above a threshold value (typically $0.15 \mathrm{~J} / \mathrm{cm}^{2}$ ) dirt ablation takes place. Between this threshold and certain fluence values that depend strongly on the substrate, the acoustic signal $S$ grows linearly as a function of the laser fluence (Fig. $5 b$ ). Also, in this region the behaviour of $S$ as a function of the number of laser shots impinging on a fixed area of the sample shows that after a certain number of shots no acoustic signal can be detected (Fig. 6 filled circles). In this fluence region microscopic inspection of the substrate after laser action shows no damage or visible surface modification. This corresponds to what we call the dirt ablation region.

However, for fluence values above the linear region, microscopic inspection of the substrate clearly shows damage produced by ablation. In these cases the laser pulse not only ablates the dirt, but parts of its energy also interacts with the substrate. We can conclude that substrate ablation occurs at higher laser fluences and can be distinguish by abrupt changes in the ratio between the sound intensity and the laser energy (Fig. 5a). These are the cases shown in Fig. 6 (open squares and triangles) for which, after the first shot, each new shot not only removes the dirt but also modifies the substrate.

We also studied the dependence of the acoustic signal, as a function of the excitation area $(A)$ in the dirt ablation region.
For that we keep the values of the fluence constant and change the diameter of the beam and the energy of the laser. In these cases linear relationships were found between $S$ and $A$.

We can conclude that acoustic detection can be used for monitoring the ablation processes real time. Typical ablation curves obtained in this way show fluence thresholds for surface dirt ablation of the order of $0.1 \mathrm{~J} / \mathrm{cm}^{2}$, and substrates ablation thresholds of at least one order of magnitude greater than this value. Then, typical fluences between $0.1 \mathrm{~J} / \mathrm{cm}^{2}<$ $F<0.5 \mathrm{~J} / \mathrm{cm}^{2}$ are best suited for the acoustic measurement of surfaces cleanliness. In this fluence region each shot of the laser ablates part of the surface dirt, and completely cleans the surface after a certain number of laser shots, as can be seen in Fig. 6.

\section{Comparison between acoustic and standard laboratory measurements}

In order to evaluate the possibilities and advantages of the new method developed in this study we compare measurements of surface cleanliness of different samples, performed by standard techniques relative to the above described acoustic ones. The standard laboratory procedure for that purpose is transmission measurements. For transparent substrates these can be made directly. In the case of opaque substrates the tape method is used.

Figure 7 shows the correlation between the acoustic measurement and the tape method for a cold rolled steel sample with dirt residuals generated during manufacturing processes. The dispersion can be attributed to the inherent uncertainties of the tape test.

Figure 8 shows the correlation for the case of candle soot deposited on glass substrates.

As can be seen in both cases linear relationships were obtained between the sound intensity and the absorbed energy. The reason that the straight lines don't pass through the origin is due to the fact that transmission measurements are sensitive, not only to absorption but also to scattering produced by the wax film that acts as a host for the soot.

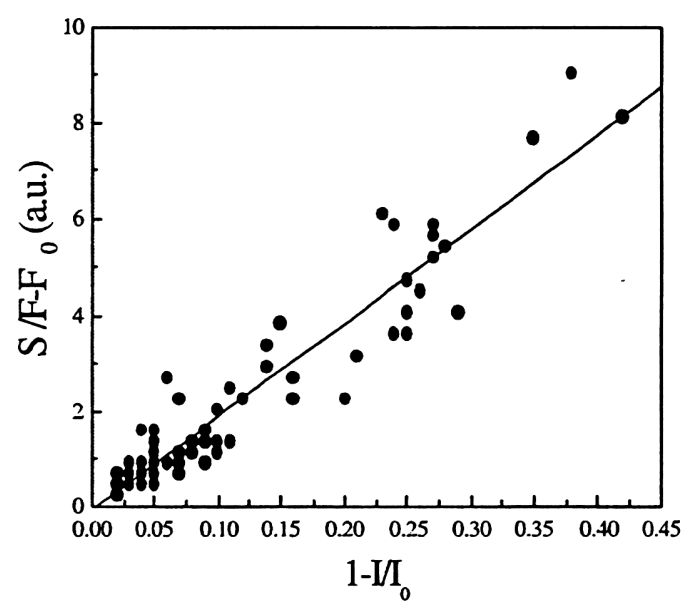

FIGURE 7 Correlation between Tape method and acoustic method. $I / I_{0}$ is the reflectivity of the tape over a white paper 


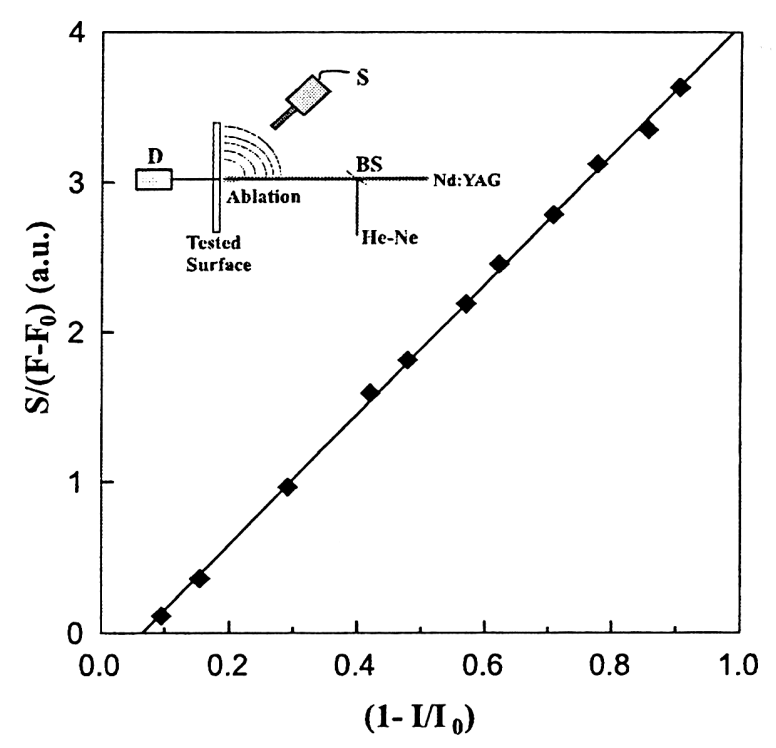

FIGURE 8 correlation between transmission measurements performed with a $\mathrm{He}-\mathrm{Ne} \mathrm{CW}$ laser and acoustic measurements for a sample of candle soot deposited on a glass substrate. Insert describes the experimental set-up used for the measurements. D: photodetector; S: microphone and BS: beam splitter

This result can be described by the following simple expression:

$S=K\left(F-F_{0}\right)\left(1-I / I_{0}\right)$.

Where $S$ is the peak to peak amplitude of the acoustic signal; $K$ is a constant that depends on the nature of the dirt film and the detector geometry and sensitivity; $F$ is the fluence; $F_{0}$ is the threshold fluence for dirt ablation; $I$ the light transmitted intensity by the tape or the substrate plus dirt, and $I_{0}$, the light transmitted intensity of the tape or the substrate without dirt.

The transmission coefficient $I / I_{0}$ contains the information on the particle concentration through the Beer's law:

$I / I_{0}=e^{-\sigma \mathrm{Nd}}=e^{-\sigma \eta_{\mathrm{s}}}$,

where $\sigma$ is the average particle cross section, $N$ the particle concentration, $d$ the thickness and $\eta_{s}$ the particle surface density

\subsection{Characterization of dirt reference samples}

Figures 9 and 10 shows the dependence of the peak to peak amplitude of the acoustic signal $S$ as a function of the laser fluence for references samples made by printing a homogeneous distribution of dots (Point diameter $\Phi=60 \mu \mathrm{m}$ and $\Phi=110 \mu \mathrm{m}$ ) of different point density on white paper, as previously described. A linear relationship $S$ vs. $F$ was observed for all the samples. Threshold fluences $F_{0}=0.08 \mathrm{~J} / \mathrm{cm}^{2}$ for $\Phi \equiv 60 \mu \mathrm{m}$ and $F_{0} \equiv 0.13 \mathrm{~J} / \mathrm{cm}^{2}$ for $\Phi=110 \mu \mathrm{m}$ were determined. Due to limitations in the printing procedure for each point diameter there is a limit to the dot densities that can be used. Above this limit, partial overlapping of points can be produced, and no linear relationship $S$ vs. $F$ is obtained. For the case of $\Phi=60 \mu \mathrm{m}$ dot diameters, limit point densities of $12800 \mathrm{p} / \mathrm{inch}^{2}$ were obtained. For dot diameters

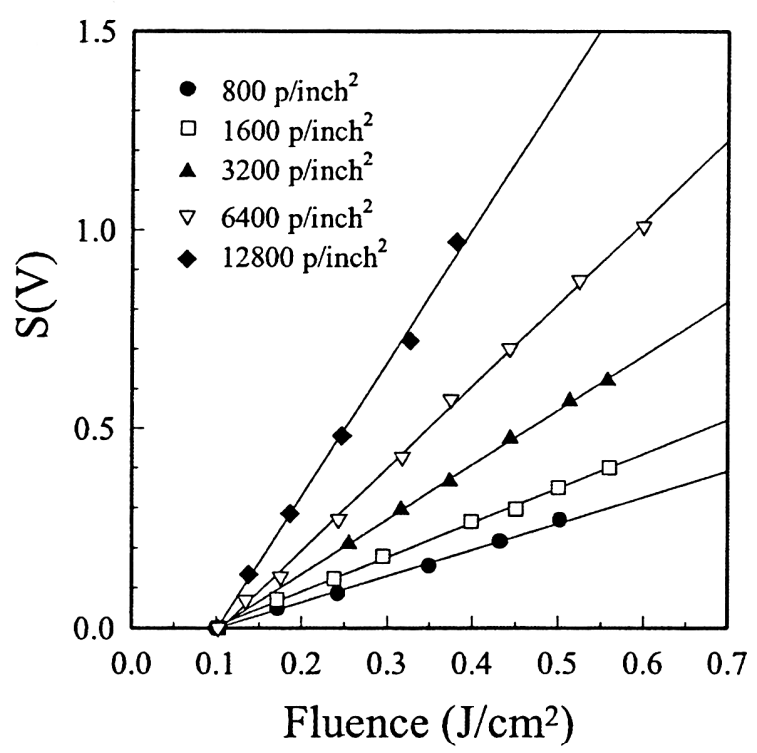

FIGURE 9 Peak to peak amplitude of the acoustic signal $S$ as a function of the laser fluence $F$ for five reference samples made by printing a homogeneous distribution of points of different points density on white paper. Point diameter $\Phi=60 \pm 10 \mu \mathrm{m}$. Threshold fluence $F_{0}=0.08 \mathrm{~J} / \mathrm{cm}^{2}$

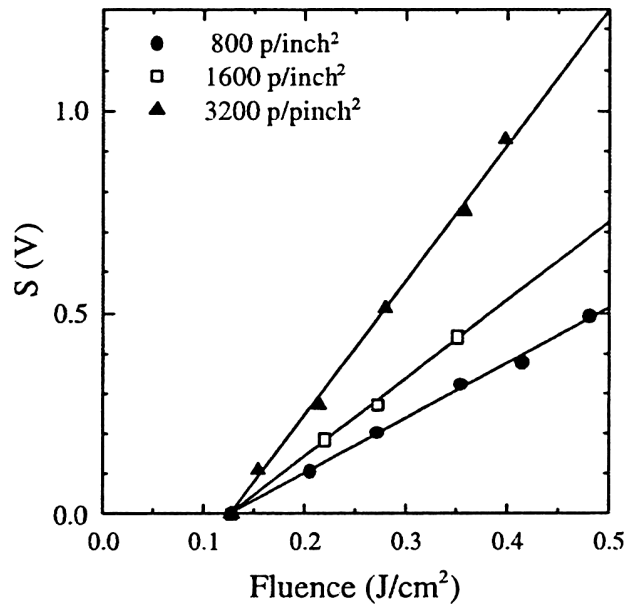

FIGURE 10 Peak to peak amplitude of the acoustic signal $S$ as a function of the laser fluence $F$ for four reference samples made by printing a homogeneous distribution of points of different point density on white paper. Point diameter $\Phi=110 \pm 10 \mu \mathrm{m}$. Threshold fluence $F_{0}=0.13 \mathrm{~J} / \mathrm{cm}^{2}$

of $110 \mu \mathrm{m}$, the limit point densities for linear behavior were $3200 \mathrm{p} /$ inch $^{2}$.

For this case (1) and (2) can be used assuming $\sigma=A$ (the dots are opaque), where $A$ is the point surface. Figure 11 shows the dependence of $S /\left(F-F_{0}\right)$, obtained from the slopes of Figs. 9 and 10, as a function of the point coverage (ie. "dirt absorption") $1-e^{-\sigma \eta_{s}}$.

As can be seen from Fig. 11, a linear relationship between the fluence normalized acoustic signal and the dirt absorption was found. The interception at the origin of the corresponding straight line can be interpreted as a constant background signal $\left(S_{0}\right)$ : The origin of this signal can be explained by the fact that the paper that acts as a substrate for the printed dots is not completely "clean". Small points produced by the splash of the ink are randomly distributed on the white paper, and dur- 


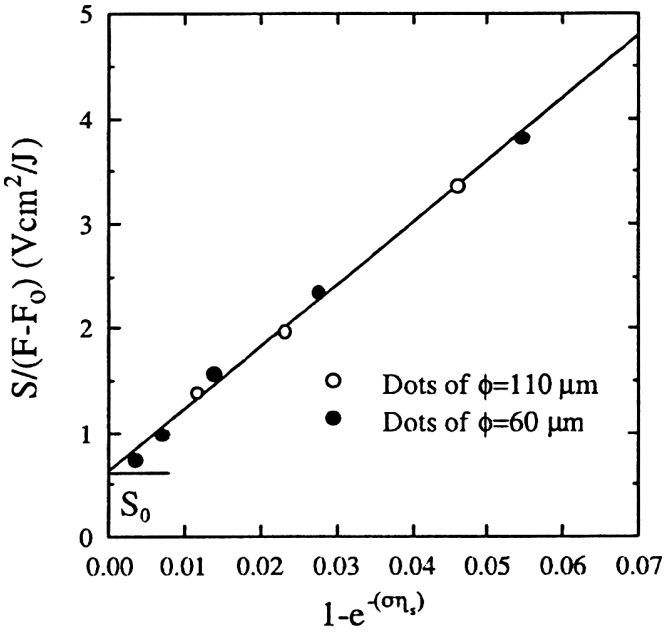

FIGURE 11 Normalized acoustic signal obtained from the slopes of Figs. 9 and 10 as a function of the point coverage

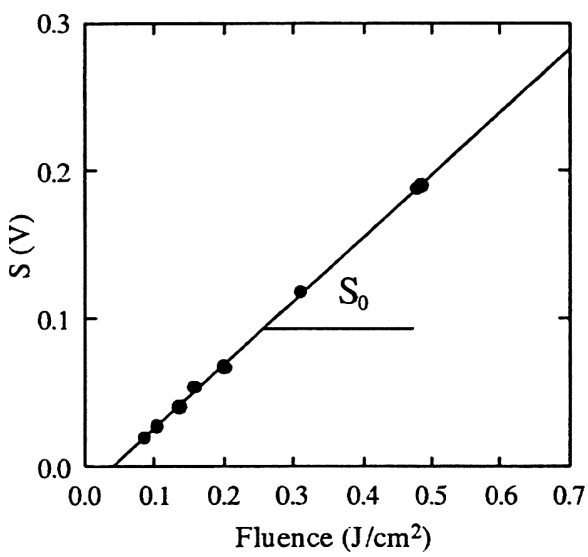

FIGURE 12 Acoustic signal $S_{0}$ vs. fluence $F$ of a non-printed region of the dirt reference (white paper printed with dots). The slope represents the background signal, and corresponds to the ordinate interception value of Fig. 11

ing ablation it causes a contribution to the signal produced by the printed distribution of points.

Figure 12 shows the determination of this contribution $S_{0}$, performed by measuring the peak to peak amplitude of $S$ as a function of $F$ in a region of the substrate paper without printed dots. As can be seen, the slope of the linear relationship found for $S$ vs. $F\left(0.45 \mathrm{Vcm}^{2} / \mathrm{J}\right)$ agrees with the ordinate value of Fig. 11. Then, $S_{0}$ must be subtracted from $S$ each time that the reference sample is used.

\section{Conclusions}

We developed a new method for real time measurement of the degree of cleanliness or the amount of dirt of any surface. The method is based on the detection of the peak- to-peak amplitude of the acoustic signal generated after laser ablation of dirt. It overcomes the most important drawbacks of standard techniques improving quality and reliability, allowing quality control and the possibility of immediate correction of processing parameters that give rise to inadequate cleanliness of the tested material. Measurements can be performed in real time and automatically, avoiding uncertainties arising from manual and laboratory operation, required in standard methods. Due to the speed of the technique, multiple measurements allow the determination not only of the average dirt content but also its standard deviation in one or different regions of the surface sample. A distribution map of the main dirt can then be obtained.

We also presented a standard reference of dirt that uses printed dots on white paper, of known size and density. This reference can be used for calibration purposes and to study, in a controlled way, the physical bases of the method.

Finally, an analytical expression was found for the acoustic signal generated after laser ablation, as a function of the laser fluence and the surface dirt parameters.

\section{REFERENCES}

1 T. Berdinskikh, H. Tkalec, Photonics Spectra, 38, 66 (2004)

2 E.Y. Assendel'ft, V.I. Beklemyshev, I.I. Makhonin, N.Y. Petrov, A.M. Prokhorov, V.I. Pustovoy, Sov. Tech. Phys. Lett. 14(6), 444 (1988)

3 A.C. Tam, W.P. Leung, W. Zapka, W. Ziemlich, J. Appl. Phys. 71, 3515 (1992)

4 Y.F. Lu, W.D. Song, B.W. Ang, D.S.H. Chan, T.S. Low, Appl. Phys. A 65, 9 (1997)

5 V. Zafiropulos, C. Fotakis, Lasers in the conservation of painted artworks, Chap. 6 In: Laser in Conservation: an introduction, ed. by M. Cooper (Butterworth Heineman, Oxford 1998)

6 Y.W. Zheng, B.S. Luk'yanchuk, Y.F. Lu, W.D. Song, Z.H. May, J. Appl. Phys. 90, 2135 (2001)

7 B. Luk'yanchuk (Ed.), Laser Cleaning and references therein. In: $O p$ tical Physics, Applied Physics and Material Science (World Scientific 2002)

8 Ford Specification, "Determination of carbon contamination on coldrolled steel sheets", No ESB-M2P117-A. The Sumitomo Search 30 (1985)

9 European Commission EUR 18634. Rolling oil distillation and thermocracking reactions in $\mathrm{HNX}$ and $100 \% \mathrm{H}_{2}$ annealing, technical steel research series, (1996)

10 Method for determining the surface cleanliness with infrared spectroscopy. Patent application. International Appl. Number: WO2004/061438 Boeing Company, US, (2004)

11 Pressure-sensitive tape method. ISO 8502-3/92. International Organization for Standarization. www.iso.org (1992)

12 G.M. Bilmes, O.E. Martínez, método para la determinaciónde suciedad en superficies. Patent AR990627, Argentina (1999)

13 O.E. Martinez, G.M. Bilmes, D.J.O. Orzi, P. Seré, R. Musso, A. Pignotti, Surf. Eng. 18, 67 (2002)

14 G.M. Bilmes, O.E. Martínez, P. Seré, R. Musso, D.J.O. Orzi,A. Pignotti, Lat. Amer. Appl. Res. 32, 263 (2002)

15 G.M. Bilmes, O.E. Martínez, Equipo láser para la medición de suciedad en chapas de acero. Patent P00010124, Argentina (2000)

16 G.M. Bilmes, O.E. Martínez, Laser Apparatus for measuring dirt density on steel plates. Patent 6.546.784-EEUU (2003)

17 D.J.O. Orzi, G.M. Bilmes, Appl. Spec. 58, 1475 (2004) 